\title{
Star Captures by Quasar Accretion Disks: A Possible Explanation of the $M-\sigma$ Relation
}

\author{
Jordi Miralda-Escudé ${ }^{1,2} \&$ Juna A. Kollmeier ${ }^{1}$ \\ 1 The Ohio State University \\ ${ }^{2}$ Institut d'Estudis Espacials de Catalunya/ICREA \\ jordi@astronomy.ohio-state.edu, jak@astronomy.ohio-state.edu
}

\begin{abstract}
A new theory of quasars is presented in which the matter of thin accretion disks around black holes is supplied by stars that plunge through the disk. Stars in the central part of the host galaxy are randomly perturbed to highly radial orbits, and as they repeatedly cross the disk they lose orbital energy by drag, eventually merging into the disk. Requiring the rate of stellar mass capture to equal the mass accretion rate into the black hole, a relation between the black hole mass and the stellar velocity dispersion is predicted of the form $M_{B H} \propto \sigma_{*}^{30 / 7}$. The normalization depends on various uncertain parameters such as the disk viscosity, but is consistent with observation for reasonable assumptions. We show that a seed central black hole in a newly formed stellar system can grow at the Eddington rate up to this predicted mass via stellar captures by the accretion disk. Once this mass is reached, star captures are insufficient to maintain an Eddington accretion rate, and the quasar may naturally turn off as the accretion switches to a low-efficiency advection mode. The model provides a mechanism to deliver mass to the accretion disk at small radius, probably solving the problem of gravitational instability to star formation in the disk at large radius. We note that the matter from stars that is incorporated to the disk has an average specific angular momentum that is very small or opposite to that of the disk, and discuss how a rotating disk may be maintained as it captures this matter if a small fraction of the accreted mass comes from stellar winds that form a disk extending to larger radius. We propose several observational tests and consequences of this theory.
\end{abstract}

Subject headings: black holes - quasars: formation - galaxies: formation - galaxies: nuclei 


\section{Introduction}

The basic model for how quasars are able to emit their prodigious radiative luminosities from a very small region of space has been in place for a long time (Lynden-Bell 1969): a massive black hole in the center of a galaxy accretes from a thin gaseous disk, converting $\sim 10 \%$ of the rest-mass of the gas into the radiation that is emitted. The gas in the disk is heated by viscous processes as it accretes, providing energy for radiating the continuum optical-ultraviolet emission from the hot, optically thick surface of the disk (Shakura \& Sunyaev 1973; Pringle 1981).

Black holes have now been detected in the centers of many galaxies and found to correlate strongly with the presence of a spheroidal stellar component, either the bulge of a spiral galaxy or an elliptical galaxy (e.g., Kormendy \& Richstone 1995; Richstone et al. 1998, Magorrian et al. 1998). The mean mass density in the universe of nuclear black holes (i.e., black holes found in galactic nuclei and presumed to be responsible for galactic nuclear activity such as the quasar phenomenon), $\rho_{B H}$, should be related to the integrated emission from all active galactic nuclei over the past history of the universe according to

$$
\frac{\epsilon}{1-\epsilon} \rho_{B H} c^{2}=\int e(z)(1+z) d z
$$

where $e(z) d z$ is the present energy density in radiation coming from AGN in the redshift range $z$ to $z+d z$, and $\epsilon$ is the mean radiative efficiency of accretion (Soltan 1982). This relation is consistent with present observations with $\epsilon \simeq 0.1$ (e.g., Barger et al. 2001; Aller \& Richstone 2002; Yu \& Tremaine 2002; Haehnelt 2003), implying that accretion of gas from thin disks likely played the dominant role in the growth of nuclear black holes. In addition, observations have shown that the black hole mass, $M_{B H}$, is tightly related to the stellar velocity dispersion, $\sigma_{*}$, as $M_{B H} \propto \sigma_{*}^{a}$, where $a$ is in the range 4 to 4.5 (Gebhardt et al. 2000; Merritt \& Ferrarese 2000, 2001; Tremaine et al. 2002). This relation suggests that there is some connection between the accretion activity of the black hole (which determines its final mass) and the stellar system that surrounds it.

The formation and growth of nuclear black holes from an accretion disk poses a number of outstanding problems: how is the large amount of mass that must be fed to the black hole funneled from the typical sizes of galaxy spheroids $(\sim 1 \mathrm{kpc})$ into the tiny region in the center of a galaxy where the mass is dominated by the central black hole $(\sim 1 \mathrm{pc})$, and into the inner accretion disk where most of the energy of active galactic nuclei is radiated $\left(\sim 10^{-2}\right.$ to $\left.10^{-5} \mathrm{pc}\right)$ ? What prevents this gas from turning into stars well before coming close to the central accretion disk, as normally occurs in galactic gaseous disks and in galaxies with an irregular distribution of gas? Once the gas is in the accretion disk, what happens when the disk becomes self-gravitating in its outer parts (e.g., Shlosman, \& Begelman 1987; Goodman 
\& Tan 2003), and hence unstable to form stars? Why is the black hole mass tightly related to the velocity dispersion of the stellar system around it, when the physical scales of these components are so vastly different?

Another possibility for the growth of black holes is by the direct capture of stars that are randomly perturbed into high-eccentricity orbits. Zhao, Haehnelt, \& Rees (2002) found that this mechanism leads to a relation $M_{B H} \simeq 10^{8} M_{\odot}\left(\sigma / 200 \mathrm{~km} \mathrm{~s}^{-1}\right)^{5}\left(t_{0} / 14 \mathrm{Gyr}\right)$, where $t_{0}$ is the total time during which stars can be captured by the black hole. This is valid if one makes the approximation of a full loss-cylinder ${ }^{1}$, i.e., one assumes that when stars are captured their orbits are immediately replenished by relaxation processes. The slope of this relation is in good agreement with the observations (see also Merritt \& Poon 2003). However, in this model black holes do not grow by gas accretion, so the similarity of the black hole mass density and the energy density from quasars at present is not accounted for. In addition, black holes would grow over a timescale too long to account for their presence at the high redshifts at which quasars are observed. Moreover, the relation $M_{B H} \propto \sigma_{*}^{5}$ holds only for black hole masses $M_{B H} \gtrsim 10^{8} M_{\odot}$, which can swallow normal stars whole without tidally disrupting them, as discussed by Zhao et al. (2002).

We propose a different idea in this paper. Black holes grow during the quasar epoch by accreting gas from a thin accretion disk in the standard way. At the same time, stars from the stellar system around the black hole are captured into the accretion disk when their orbits become highly eccentric and they plunge through the disk. Even though the stars are slowed down by only a small fraction of their velocity due to the drag force at every disk crossing, repeated crossings result in their final merger into the disk. The capture of stars by an accretion disk, discussed by Ostriker (1983), Syer, Clarke, \& Rees (1991), Artymowicz et al. (1993), and Zurek et al. (1994), can have a much higher cross section than direct capture by the black hole, shortening the time required for the black hole to grow. The growth of black holes from a gaseous disk that is continuously replenished with matter from plunging stars can also solve the problem of how matter is transported from the galactic system to the very small central accretion disk, and provides a way to connect the final mass of the black hole after accretion stops with the velocity dispersion of the surrounding stellar system.

Delivering mass to the accretion disk by means of stars that are randomly scattered into the loss-cylinder implies that the structure of the disk should change in an important way owing to the addition of energy and angular momentum to the disk as the plunging stars

\footnotetext{
${ }^{1}$ We use the term loss-cylinder to refer to the region of phase-space from which stars will be captured. At a fixed point in space in a spherical potential, the shape of this region in velocity space is a narrow cylinder along the radial velocity axis. This has usually been referred to as loss-cone in the literature.
} 
dissipate their kinetic energy during disk crossings and the angular momentum of the stars is incorporated to the disk. The issue of the global angular momentum will be discussed in $\S 5$. In this paper, we will generally assume a steady-state disk structure ignoring the effects of the stars, leaving for later work a fully self-consistent model in which the effect of the plunging stars added to the disk is taken into account.

The model is presented in detail in $\S 2$, where the condition for stars to be captured by the disk is described and a resulting $M_{B H}-\sigma$ relation is inferred. In $\S 3$ we discuss how the generic problem of self-gravity of the disk may be solved in our model, and in $\S 4$ we comment on the fate of the stars after they are embedded in the disk. $\$ 5$ describes the total disk angular momentum problem and proposes a solution. Finally, $\S 6$ discusses the predictions of the model and presents the conclusions.

\section{Black Hole Growth from Collisions of Stars with the Accretion Disk}

Before going into the detailed description of our model, it will be useful to give an overview of our goals in this section. We will start by reviewing steady-state accretion disk models, deriving the surface density profile. We will then infer the condition required for a typical main-sequence star to be captured by the disk as it slows down in multiple disk crossings. As we shall show below, captured stars are eventually destroyed and their matter is dispersed within the disk. This will lead us to a rate at which mass is being delivered to the disk by the plunging stars. We will then require that this mass delivery rate from stars into the disk is equal to the mass accretion rate of the disk gas into the black hole, which is given in terms of the quasar luminosity and the efficiency at which the accreted mass is converted to radiation. This requirement ensures that the disk can remain in a steady state, capturing the mass it needs to continue fueling the black hole, and will lead to a relation between the black hole mass and the velocity dispersion of the stellar system.

This still leaves two remaining questions: how the accretion disk is initially started so that it can gain matter by capturing stars, and when the mechanism of stellar captures and black hole growth stops. We will propose that when a starburst takes place in a galactic nucleus, a small seed black hole with an initial accretion disk (made, for example, by tidally disrupted stars) naturally grows its accretion disk by capturing stars, and maintains it at the level required to accrete and shine as a quasar with a luminosity near the Eddington value. The black hole grows in mass until it reaches the value determined by our derived relation with the velocity dispersion of the stars that were formed around the black hole. After this value is reached, the rate of star captures is too small to maintain an accretion rate near the Eddington value. This reduces the surface density of the disk, further decreasing the star 
capture rate until, perhaps when accretion switches from a thin disk to an advection mode, the quasar turns off and leaves the black hole at a fixed predetermined mass. This can then

explain the observed $M_{B H}-\sigma_{*}$ relation of the present remnant black holes with the velocity dispersion of the spheroidal systems around them.

We start by summarizing the standard steady-state thin accretion disk model, transporting angular momentum by an effective viscosity as described in the usual $\alpha$-model (Shakura \& Sunyaev 1973; Pringle 1981). We closely follow Goodman (2003), reproducing some equations here for completeness. In order to determine whether a star that plunges through the disk can be captured, we must first obtain the disk surface density profile.

\subsection{The Surface Density Profile of Steady Accretion Disks}

Matter in an accretion disk can move in towards the black hole as angular momentum is transported out by viscous processes. We assume a steady-state accretion disk of surface density $\Sigma(r)$ at radius $r$ and angular rotation rate $\Omega=\left(G M_{B H} / r^{3}\right)^{1 / 2}$, with a constant accretion rate $\dot{M}$, where viscosity causes the gas to drift inward at a radial speed $v_{r}(r)$ which is much smaller than the tangential orbital velocity $\Omega r$. The viscosity force per unit area can be imagined as acting on the surface of a cylinder at radius $r$ that cuts the disk vertically, and is equal to $\nu \rho r \Omega^{\prime}$, where $\nu$ is the viscosity coefficient, $\rho$ is the density, and $\Omega^{\prime}=d \Omega / d r$. This force, integrated vertically, causes a torque on the disk inside the cylinder of $2 \pi r \nu \Sigma r^{2} \Omega^{\prime}$. Considering a ring of the disk at radius $r$, the net torque acting on the ring per unit ring width $d r$ is $d\left(2 \pi \nu \Sigma r^{3} \Omega^{\prime}\right) / d r$. The angular momentum of the ring per unit ring width is $2 \pi r \Sigma \Omega r^{2}$, and conservation of angular momentum in steady-state conditions implies

$$
\frac{d\left(\sum r^{3} \Omega v_{r}\right)}{d r}=\frac{d\left(\nu \Sigma r^{3} \Omega^{\prime}\right)}{d r}
$$

Integrating this equation, and substituting $\Omega^{\prime}=-3 \Omega /(2 r)$, and $v_{r}=-\dot{M} /(2 \pi r \Sigma)$ as required by mass conservation in steady-state, we have

$$
\nu \Sigma=\frac{\dot{M}}{3 \pi}\left[1-\left(\frac{r_{i n t}}{r}\right)^{\frac{1}{2}}\right],
$$

where $r_{\text {int }}$ is an integration constant that depends on an inner boundary condition. In practice, $r_{i n t}$ is determined by the relativistic inner regions of the accretion disk and is negligible in the outer regions that we will be interested in, so we can use

$$
\Sigma=\frac{\dot{M}}{3 \pi \nu}
$$


The viscosity is usually assumed to be related to turbulent processes, and is of the order of the product of the velocity and size of the largest turbulent eddies. Following Goodman (2003), the viscosity coefficient is expressed as $\nu=\alpha \beta^{b} c_{s}^{2} / \Omega$, where $c_{s}=\sqrt{p / \rho}$ is the isothermal sound speed at the midplane, $\rho$ is the gas density, $\beta=p_{\text {gas }} / p$, and $p_{\text {gas }}$ and $p$ are the gas and total pressure, respectively, with $p-p_{\text {gas }}=p_{\text {rad }}$ being the radiation pressure. If viscosity is produced by magnetorotational instability, the dimensionless viscosity parameter $\alpha$ is thought to be between $10^{-3}$ and $10^{-1}$ (Balbus \& Hawley 1998). When radiation pressure dominates, we assume the viscosity may be proportional to either the total pressure $(b=0)$ or to the gas pressure $(b=1)$.

Writing also $\dot{M}=L /\left(\epsilon c^{2}\right)=\dot{m} L_{E d d} / c^{2}$, where $L$ is the radiative luminosity of the disk, $\epsilon$ is the radiative efficiency, $L_{E d d}=4 \pi c G M_{B H} / \kappa_{e}$ is the Eddington luminosity, $\kappa_{e}$ is the electron scattering opacity, and the accretion rate has been conveniently parameterized as $\dot{m}=L /\left(\epsilon L_{E d d}\right)$, we obtain

$$
\dot{M}=\frac{4 \pi G M_{B H} \dot{m}}{c \kappa_{e}}
$$

and

$$
\Sigma=\frac{4 G M_{B H} \dot{m} \Omega}{3 \alpha c \kappa_{e} c_{s}^{2} \beta^{b}}
$$

To proceed further, it is necessary to specify the energy balance in the disk to compute the midplane temperature, $T$, related to the isothermal sound speed by $\beta c_{s}^{2}=\left(k_{B} T\right) /\left(\mu m_{p}\right)$, where $k_{B}$ is the Boltzmann constant and $\mu m_{p}$ is the mean particle mass. Assuming that viscous dissipation of the orbital energy is the dominant heat source, the energy dissipation rate per unit area of the disk is $\nu \Sigma\left(r \Omega^{\prime}\right)^{2}$. This must be equal to the radiative energy emitted per unit area by the two sides of the disk, $2 \sigma T_{e f f}^{4}$, where $\sigma$ is the Stefan-Boltzmann constant. Substituting $\Omega^{\prime}=-(3 / 2)\left(G M_{B H}\right)^{1 / 2} / r^{5 / 2}$ and using equations (4) and (5), one finds

$$
\sigma T_{e f f}^{4}=\frac{3 G^{2} M_{B H}^{2} \dot{m}}{2 c \kappa_{e} r^{3}}
$$

The midplane temperature can be approximated by $T^{4} \simeq \kappa(\Sigma / 2) T_{e f f}^{4}$, where $\kappa$ is the opacity. Hence,

$$
T=\left(\frac{3 G^{2} M_{B H}^{2} \dot{m} \hat{\kappa} \Sigma}{4 \sigma c r^{3}}\right)^{\frac{1}{4}}
$$

where $\hat{\kappa}=\kappa / \kappa_{e}$. Replacing the temperature by the isothermal sound speed, substituting into equation (6), and using $\sigma=\left(2 \pi^{5} k_{B}^{4}\right) /\left(15 c^{2} h^{3}\right)$, where $h$ is the Planck constant, we find

$$
\Sigma=\frac{4 \pi}{3 c}\left[\frac{2}{15 \hat{\kappa}(r)}\right]^{\frac{1}{5}}\left(\frac{\dot{m}}{h r}\right)^{\frac{3}{5}}\left[\frac{G M_{B H} \mu m_{p}}{\alpha \kappa_{e} \beta(r)^{b-1}}\right]^{\frac{4}{5}} .
$$


The quantity $\beta=p_{\text {gas }} / p$ can be expressed in terms of $\Sigma$ and $T$ using $p_{\text {gas }}=\rho k_{B} T /\left(\mu m_{p}\right)$, $p_{\text {rad }}=4 \sigma T^{4} /(3 c)$, and $\rho=\Sigma \Omega /\left(2 c_{s}\right)$ (since the disk scale height is $H=c_{s} / \Omega$ ). Using equations (8) and (9), one finds

$$
\frac{\beta^{\frac{4+b}{10}}}{1-\beta}=\frac{(45)^{\frac{1}{10}} \pi^{\frac{3}{40}}}{2^{\frac{1}{20}}}\left(\frac{m_{P l}^{2} m_{e}^{2}}{\alpha \alpha_{e}^{2} \mu^{4} m_{p}^{3} M_{B H} \hat{\kappa}^{9}}\right)^{\frac{1}{10}} \dot{m}^{\frac{-4}{5}}\left(\frac{r}{R_{S}}\right)^{\frac{21}{20}} .
$$

Here, we have used $\kappa_{e}=4 \pi \alpha_{e}^{2} \hbar^{2}(1+X) /\left(3 m_{p} m_{e}^{2} c^{2}\right)$, where $\hbar=h /(2 \pi), m_{p}$ is the proton mass, $X$ is the hydrogen abundance by mass, and $\alpha_{e}$ is the fine structure constant. We also use the Schwarzschild radius $R_{S}=2 G M_{B H} / c^{2}$, and the Planck mass $m_{P l}=\sqrt{\hbar c / G}$. For characteristic values of the black hole mass $M_{B H}=10^{8} M_{8} M_{\odot}, \alpha=10^{-2} \alpha_{-2}, X=0.7$, and $\mu=0.62$, the $\beta$ parameter is given by

$$
\frac{\beta^{\frac{4+b}{10}}}{1-\beta}=4.6 \alpha_{-2}^{-\frac{1}{10}} \hat{\kappa}^{-\frac{9}{10}} \dot{m}^{-\frac{4}{5}} M_{8}^{-\frac{1}{10}}\left(\frac{r}{10^{3} R_{S}}\right)^{\frac{21}{20}}
$$

the surface density is

$$
\Sigma=7.6 \times 10^{5}\left(\alpha_{-2} \beta^{b-1}\right)^{-\frac{4}{5}} \dot{m}^{\frac{3}{5}}\left(\frac{M_{8}}{\hat{\kappa}}\right)^{\frac{1}{5}}\left(\frac{10^{3} R_{S}}{r}\right)^{\frac{3}{5}} \mathrm{~g} \mathrm{~cm}^{-2},
$$

and the midplane temperature is

$$
T=3.9 \times 10^{4} \mathrm{~K}\left(\frac{\hat{\kappa}}{\alpha_{-2} \beta^{b-1} M_{8}}\right)^{\frac{1}{5}} \dot{m}^{\frac{2}{5}}\left(\frac{10^{3} R_{S}}{r}\right)^{\frac{9}{10}} \mathrm{~K} .
$$

We plot in Figure 1 the contours in the $M-R / R_{S}$ plane at which $\beta=0.5$, and at which the surface density and midplane temperature have the characteristic values indicated in the caption, for the case $\dot{m}=10$ and $\alpha_{-2}=1$. For all black hole masses, the pressure in the accretion disk is dominated by radiation to the left of the line $\beta=0.5$, and is dominated by matter to the right of this line. For the case $b=1, \Sigma$ is independent of $\beta$ and, assuming that $\hat{\kappa}(r) \simeq 1$ and $\alpha$ is independent of $r$, then $\Sigma(r) \propto M_{B H}^{4 / 5} / r^{3 / 5}$. This form of the surface density profile will be important later for our $M_{B H}-\sigma_{*}$ relation. We also show the contour for which the opacity due to bound-free transitions is $\kappa_{b f}=\kappa_{e}$ (implying $\hat{\kappa}=2$ if other opacity mechanisms are negligible). To the left of this line we have $\hat{\kappa} \simeq 1$.

\subsection{Changes of Velocity, Orbital Energy and Mass of a Plunging Star}

The orbital angular momentum of a star in the stellar system around the black hole may be changed by relaxation processes or a triaxial potential (due to the gravitational 
contribution of stars and the gas disk), and if by chance the orbit becomes very eccentric the star may collide with the dense central part of the accretion disk. The gas drag force during the collision will then slow down the star by a velocity increment $\Delta v$, resulting in a loss of orbital energy and a corresponding reduction of the orbital apocenter and period. If the apocenter reduction is substantial enough, the star will be condemned to plunge repeatedly through the disk until its orbit is brought into the disk plane and circularized (Syer et al. 1991), leaving the star immersed inside the disk. This defines an effective loss-cylinder for the capture of a star by the disk. We now calculate the condition for the star to be captured.

As we shall see later, the typical radius at which stars are captured by crossing the disk is $r \sim 10^{3} R_{S}$, where $R_{S}$ is the Schwarzschild radius of the black hole. The orbital velocity of the star as it crosses the disk at this radius is $\sim 10^{4} \mathrm{~km} \mathrm{~s}^{-1}$, which is highly supersonic and much greater than the escape velocity from the surface of the star. This means that gravitational focusing is negligible, and hence the response of the disk to the passage of the star can be ignored for the purpose of computing the velocity change of the star $\Delta v$. Under these conditions we assume, as a first approximation, that $\Delta v$ is simply determined by the absorption of the momentum of all the disk gas that lies along the path of the star:

$$
\Delta v=\frac{\Sigma}{\Sigma_{*}} \frac{v}{\sin \theta}
$$

where $\Sigma_{*}=M_{*} /\left(\pi R_{*}^{2}\right)$ is the mean surface density of the star of mass $M_{*}$ and radius $R_{*}, \theta$ is the angle between the orbital velocity of the star and the plane of the disk at the intersection point, and $v$ is the relative velocity between the star and the disk gas moving on a circular orbit. Typically, $\Sigma_{*} \sim 10^{11} \mathrm{~g} \mathrm{~cm}^{-2}$ and $\Sigma \sim 10^{6} \mathrm{~g} \mathrm{~cm}^{-2}$ (see Fig. 1 ), so $\Delta v / v \sim 10^{-5}$ for each star passage through the disk.

Apart from reducing their velocity, stars may also lose a small fraction of their mass at every disk passage. As will be discussed below in $\S 4$, stars may survive essentially intact through many disk passages until they merge inside the disk, but after this they should be destroyed and their matter should dissolve into the disk.

A complete analysis of the rate at which stars are captured by the disk would require computing the probability distribution of $\Delta v$ from a random distribution of orbital inclinations and pericenter longitudes (see Ostriker 1983 for a calculation along this line to compute the rate of angular momentum loss of the disk by star crossings). In this paper we make a more approximate estimate based on considering a typical star orbit. We use $v \simeq\left(2 G M_{B H} / r\right)^{1 / 2}$ (neglecting the circular velocity of the gas in the disk), and assume the case where the star crosses the disk at its pericenter. The average value of $1 / \sin \theta$, where $\theta$ is the angle between the plane of the orbit and the plane of the disk (with probability 
distribution $\sin \theta d \theta$ ), is then $\pi / 2$, so the change in the orbital energy per unit mass is

$$
\Delta E=v \Delta v=\frac{\Sigma}{\Sigma_{*}} \frac{\pi G M_{B H}}{r_{p}},
$$

where $r_{p}$ is the pericenter. Averaging over all possible pericenter longitudes would not greatly modify this result (for example, one can easily show that for a pericenter longitude of 90 degrees, the collision of the star with the disk takes place at a radius $2 r_{p}$, which reduces the disk surface density by $2^{-3 / 5}$ and the square of the relative velocity by $1 / 2$, but then $\Delta v$ is increased by a factor 2 because there are two equal collisions per orbit and by another factor $\sqrt{2}$ because the sine of the angle between the disk plane and the stellar velocity is smaller by a factor $\sqrt{2}$ ).

\subsection{The Size of the Region from which Stars are Captured}

We now consider that we have a stellar system that is roughly isotropic in the central region of the galaxy where the gravitational force is dominated by the nuclear black hole. Among all orbits with a semimajor axis $a$, the probability distribution of the orbital pericenter $r_{p}$ is $2\left(1-r_{p} / a\right) d r_{p} / a$ (because an isotropic system in a point-mass potential has a flat distribution in the square of the eccentricity). Hence, if the time during which the gas disk is maintained with a roughly constant surface density is $t_{S}$ (which we identify later with the Salpeter time for the growth of a black hole, see Salpeter 1964), the number of times that a star will cross the disk at a pericenter smaller than $r_{p}$ is $N_{\text {orb }}=\left(t_{S} / P\right)\left(r_{p} / a\right)\left(2-r_{p} / a\right)$, where $P=2 \pi a^{3 / 2} /\left(G M_{B H}\right)^{1 / 2}$ is the period. Using equation (15), the average rate of orbital energy loss of a star with semimajor axis $a$ due to passages at all pericenters $r_{p}$ is

$$
\frac{d E}{d t}=\int_{r_{1}}^{a} \frac{d r_{p}}{a P} 2\left(1-\frac{r_{p}}{a}\right) \frac{\Sigma\left(r_{p}\right)}{\Sigma_{*}} \frac{\pi G M_{B H}}{r_{p}} .
$$

Note that this integral diverges at small $r_{p}$, that is to say, the loss of orbital energy is dominated by the disk crossings closest to the center, when the eccentricity is closest to one. We choose the lower limit of the integral, $r_{1}$, to be the pericenter for which $N_{\text {orb }}=1$, because a typical star will not cross the disk at a smaller pericenter. Using the approximation $r_{1} \ll a$, this pericenter is $r_{1}=(a P) /\left(2 t_{S}\right)$. Note also that it is essential here to assume that the rate of relaxation is fast enough to keep the loss-cylinder full, so that the probability to have a star in a pericenter range $d r_{p}$ (when $r_{p} \ll a$ ) is $2 d r_{p} / a$. Assuming $\Sigma(r) \propto r^{-3 / 5}$ (which is true for $b=1$, or for the gas-pressure-dominated region of the disk if $b=0$ ), we have

$$
\frac{d E}{d t}=\frac{10}{3 P} \frac{\Sigma\left(r_{1}\right)}{\Sigma_{*}} \frac{\pi G M_{B H}}{a} .
$$


Substituting the orbital energy per unit mass in a point-mass potential, $E=G M_{B H} /(2 a)$, we find

$$
\frac{d a}{d t}=-\frac{20 \pi a}{3 P} \frac{\Sigma\left(r_{1}\right)}{\Sigma_{*}} .
$$

Assuming again $\Sigma\left(r_{1}\right) \propto r_{1}^{-3 / 5}$, and using $P \propto a^{3 / 2}$, and $r_{1} \propto a P \propto a^{5 / 2}$, we have $d a / d t \propto a^{-2}$. Hence, the condition for the star to be captured by the disk in a time less than $t_{S}$ is that, at the initial semimajor axis,

$$
\|d a / d t\|>a /\left(3 t_{S}\right) .
$$

Substituting (19) into equation (18), we find that the capture condition is that the initial semimajor axis must be smaller than a critical radius $r_{c}$ that obeys

$$
\frac{\Sigma\left(r_{1 c}\right)}{\Sigma_{*}}=\frac{P_{c}}{20 \pi t_{S}}=\frac{r_{c}^{3 / 2}}{10\left(G M_{B H}\right)^{1 / 2} t_{S}},
$$

where $P_{c}$ is the period at $r_{c}$, and $r_{1 c}=r_{c} P_{c} /\left(2 t_{S}\right)$.

Because the loss of orbital energy is dominated by disk crossings at the smallest pericenters, a typical star starting at $a \simeq r_{c}$ loses little orbital energy until by chance it enters the loss-cylinder at a pericenter $\lesssim r_{1 c}$, and then substantially reduces its semimajor axis in a single disk crossing. After that, relaxation may take the star out of the loss-cylinder if it occurs fast enough, but as $a$ decreases the timescale for randomly entering the loss-cylinder again is rapidly shortened in any case (because on average, $d a / d t \propto a^{-2}$ as shown above), so the star will inevitably be captured. The radius $r_{c}$ represents the semimajor axis for which the average time to enter the loss-cylinder for disk capture is $t_{S}$.

In practice, the rate of relaxation will not be so large to make all stars of a fixed semimajor axis have the same probability to be captured. Stars on loop orbits may never come close to the center, and only the stars that can reach the phase space region of zero angular momentum from their initial orbit with the available rate of relaxation will be captured. Provided these latter stars are a large enough fraction of the total within the radius $r_{c}$, the approximation of a full loss-cylinder is appropriate and equation (18) still gives an average rate of reduction of the semimajor axis. Some stars on loop orbits within $r_{c}$ may never reach the loss-cylinder, while other stars slightly further than $r_{c}$ may be captured if they start on highly radial orbits. We will ignore these complications here and assume that the stars that are captured are those that start with $a<r_{c}$. 


\subsection{The Black Hole Mass - Velocity Dispersion Relation}

We can now clearly see why there should be a relation between the black hole mass and velocity dispersion of the stellar system around the black hole. Assuming the stars follow an isothermal density profile, the stellar system has a mass within a radius $r_{c}$ given by its velocity dispersion $\sigma_{c}, M_{*}\left(r_{c}\right) \simeq 2 r_{c} \sigma_{c}^{2} / G$. Over the time duration $t_{S}$ of the luminous phase of a quasar during which the gaseous disk is present, stars will continuously plunge through the disk supplying it with new gas, which will then accrete into the black hole. At the end of this process, the black hole mass will be $M_{B H}=M_{*}\left(r_{c}\right)$, so $r_{c}$ is equal to the zone of influence of the black hole as its mass reaches its final value.

From equation (20), the surface density at the pericenter where stars are captured, $\Sigma\left(r_{1 c}\right)$, is proportional to the orbital period $P_{c}$, if $t_{S}$ and $\Sigma_{*}$ are constant. Since

$$
r_{1 c} \propto r_{c} P_{c} \propto r_{c}^{5 / 2} / M_{B H}^{1 / 2},
$$

and, for $b=1$ and $\hat{\kappa}=1$, we have $\Sigma(r) \propto M_{B H}^{4 / 5} / r^{3 / 5}$ [eq. (9)], we find

$$
\Sigma\left(r_{1 c}\right) \propto M_{B H}^{4 / 5} / r_{1 c}^{3 / 5} \propto M_{B H}^{11 / 10} / r_{c}^{3 / 2} \propto P_{c} \propto r_{c}^{3 / 2} / M_{B H}^{1 / 2} .
$$

This implies that $M_{B H}^{8 / 5} \propto r_{c}^{3}$, or, using $r_{c} \propto M_{B H} / \sigma_{c}^{2}$, that

$$
M_{B H} \propto \sigma_{c}^{30 / 7},
$$

in excellent agreement with the slope of the observed power-law relation (Merritt \& Ferrarese 2001, Tremaine et al. 2002).

The full predicted relation between $M_{B H}$ and $\sigma_{c}$ is easily found starting from equations (9) and (20), combined with $r_{1 c}=\pi r_{c}^{5 / 2} /\left[t_{S}\left(G M_{B H}\right)^{1 / 2}\right]$. We also use the relation $r_{c}=$ $G M_{B H} /\left(2 \sigma_{c}^{2}\right)$ for an isothermal density profile, and $t_{S}=\left(c \kappa_{e}\right) /(4 \pi G \dot{m})$ for the Salpeter time. The result is

$$
M_{B H}=M_{*}^{\frac{5}{7}} m_{P l}^{\frac{2}{7}}\left(\frac{\alpha_{e} m_{P l}}{m_{e}}\right)^{\frac{8}{7}}\left(\frac{2 \sigma_{c}}{v_{e *}}\right)^{\frac{30}{7}}\left(\frac{v_{e *}}{3 c}\right)^{\frac{10}{7}} \dot{m}^{-\frac{5}{7}} \hat{\kappa}\left(r_{1 c}\right)^{-\frac{1}{7}}\left[\frac{5 \mu(1+X)}{\alpha \beta\left(r_{1 c}\right)^{b-1}}\right]^{\frac{4}{7}},
$$

where $m_{P l}=(\hbar c / G)^{1 / 2}$ is the Planck mass and $v_{e *}=\left(2 G M_{*} / R_{*}\right)^{1 / 2}$ is the escape velocity of the star. In terms of typical values of $M_{B H}$ and $\sigma_{c}$, this is

$$
M_{B H}=3.3 \times 10^{8} M_{\odot}\left(\frac{M_{*}}{M_{\odot}}\right)^{\frac{5}{7}}\left(\frac{\sigma_{c}}{200 \mathrm{~km} \mathrm{~s}^{-1}}\right)^{\frac{30}{7}}\left(\frac{v_{e \odot}}{v_{e *}}\right)^{\frac{20}{7}} \dot{m}^{-\frac{5}{7}} \hat{\kappa}\left(r_{1 c}\right)^{-\frac{1}{7}}\left[\alpha_{-2} \beta\left(r_{1 c}\right)^{b-1}\right]^{-\frac{4}{7}},
$$

where we have used $X=0.7$ and $\mu=0.62$. 
The normalization of the relation is also reasonably close to the observed relation $M_{B H}=$ $1.3 \times 10^{8} M_{\odot}\left(\sigma_{*} / 200 \mathrm{~km} \mathrm{~s}^{-1}\right)^{a}$ (Merritt \& Ferrarese 2001, Tremaine et al. 2002), but it is subject to many uncertainties depending on various parameters: the type of star that is most abundant in the stellar system, the viscosity parameter $\alpha$, the normalized accretion rate $\dot{m}$, and the relationship of the velocity dispersion $\sigma_{c}$ of the nuclear starburst region at the time the quasar was active to the present central velocity dispersion of the galaxy that contains the remnant black hole. On this last point, it must be realised that the stellar density we have assumed at radius $r_{c}$ is much higher than the present stellar density in observed ellipticals (for example, for $\sigma_{c}=200 \mathrm{~km} \mathrm{~s}^{-1}$ and $M_{B H} \sim 10^{8} M_{\odot}, r_{c} \simeq 5 \mathrm{pc}$, and the density profiles of observed ellipticals are shallower than isothermal up to radii much larger than $r_{c}$ ). This implies that in our model the central stellar density must have been greatly reduced after the quasar epoch due to, for example, mass loss from supernovae and winds with subsequent adiabatic expansion (in particular if the stellar mass function in nuclear starbursts is dominated by massive stars), and mergers of nuclear black holes as their host elliptical galaxies merge (Quinlan 1996; Faber et al. 1997; Milosavljević \& Merritt 2001). These processes may have altered the central velocity dispersion of the galaxy.

The quantities $\hat{\kappa}\left(r_{1 c}\right)$ and $\beta\left(r_{1 c}\right)^{b-1}$ can affect not only the normalization but also the shape of the $M_{B H}-\sigma_{c}$ relation. In Figure 1, the value of $r_{1 c}$ as a function of $M_{B H}$ is plotted (assuming $\hat{\kappa}=1$ ). This is computed by using $r_{1 c}=r_{c} P_{c} /\left(2 t_{S}\right)$, which yields:

$$
r_{1 c}=340 R_{S} \dot{m}^{\frac{1}{6}}\left(\frac{M_{*}}{M_{\odot}}\right)^{\frac{5}{6}}\left(\frac{v_{e \odot}}{v_{e *}}\right)^{\frac{10}{3}}\left(M_{8} \hat{\kappa}\right)^{-\frac{1}{6}}\left(\alpha_{-2} \beta^{b-1}\right)^{\frac{2}{3}} .
$$

As we can see, the region where $\hat{\kappa}$ exceeds unity substantially is at radius larger than $r_{1 c}$, but the disk radiation pressure can be important near $r_{1 c}$. This implies that the relation $M_{B H} \propto \sigma_{*}^{30 / 7}$ is only valid for $b=1$, that is to say, when the disk viscosity is proportional to the gas pressure. If $b=0$, and in the radiation-dominated pressure limit of $\beta \ll 1$, we can find by using equations (11), (25), and (26) that $M_{B H} \propto \sigma_{c}^{10 / 3}$. Hence, the predicted $M_{B H}-\sigma_{c}$ relation depends on details of the disk viscosity.

Generally, the $M_{B H}-\sigma_{c}$ relation in (25) depends on the disk surface density profile, which is subject to other possible modifications in addition to the viscosity mechanism. The effects of the stars crossing the disk on the surface density profile will be briefly discussed in $\S 5$. The simple thin disk model may also modified when the luminosity is approximately equal to the Eddington luminosity, and the disk thickens by the radiation pressure (Wang et al. 1999; Ohsuga et al. 2002). 


\subsection{The Evolution of a Nuclear Black Hole after the Formation of a Galactic Spheroid}

The mass-velocity dispersion relation we have derived in equation (24) originates from the condition that the rate at which new mass is delivered to the disk by plunging stars is the same as the rate at which the gas in the disk is accreted by the black hole. How does that establish the black hole mass-velocity dispersion relation in the remnant galaxies after quasars turn off?

Mergers of galaxies rich in cold gas may often give rise to strong nuclear starbursts. A seed black hole may be present in the nucleus, probably coming from one of the galaxies that merged. The seed black hole mass can initially be very small compared to the value implied by equation (24) for the velocity dispersion of the newly formed galactic spheroid. A small seed accretion disk can initially form around this black hole by a variety of mechanisms, such as residual gas left over from star formation or from stellar winds that reaches the center directly, physical stellar collisions, and tidal disruptions of stars by the black hole. This disk can then start growing and feeding the black hole by capturing stars. We now show that the capture rate of stars will be more than sufficient to maintain an Eddington accretion rate as long as the black hole mass is below the $M_{B H}-\sigma_{c}$ relation in (24).

For fixed $\sigma_{c}$ and a singular isothermal profile of stars, the black hole needs to capture stars out to a radius $r_{c} \propto M_{B H}$ to increase its own mass. Let $\mathrm{t}$ be the timescale over which these stars with total mass $M_{B H}$ are captured. Then, as the black hole grows the capture pericenter must increase as $r_{1 c} \propto r_{c} P_{c} / t \propto M_{B H}^{2} / t$ (where the orbital period at $r_{c}$ is $\left.P_{c} \propto r_{c}^{3 / 2} / M_{B H}^{1 / 2} \propto M_{B H}\right)$. By the same arguments as in the previous subsections, capturing a star over one orbit requires $\Sigma\left(r_{1 c}\right) / \Sigma_{*} \propto r_{1 c} / r_{c} \propto M_{B H} / t$. At the same time, if accretion of the disk gas to the black hole also occurs at the rate $\dot{M} \sim M_{B H} / t$ (so the normalized accretion rate is $\dot{m} \propto t^{-1}$ ), then equation (9) implies that the surface density at the capture radius is $\Sigma\left(r_{1 c}\right) \propto M_{B H}^{4 / 5} \dot{m}^{3 / 5} / r_{1 c}^{3 / 5} \propto M_{B H}^{-2 / 5}$, independent of $t$. Combining these two scalings

for $\Sigma\left(r_{1 c}\right)$, we infer $t \propto M_{B H}^{7 / 5}$. Equivalently, the normalized accretion rate is $\dot{m} \propto M_{B H}^{-7 / 5}$. This makes it clear that the lower the mass of the black hole, the shorter the time required for the gas disk to capture stars with a mass equal to that of the black hole. And viceversa, if the black hole exceeds the mass in equation (24), then the gas disk must take longer than the time $t_{S}$ to capture stars with a total mass $M_{B H}$.

We note that our inferred proportionality $\dot{m} \propto M_{B H}^{-7 / 5}$ when the disk is capturing stars from a stellar cluster with fixed $\sigma_{c}$ implies that the accretion rate is super-Eddington when the black hole mass is small. Under these circumstances, a thin disk with the surface density profile (9) is probably not valid (although disk models with super-Eddington accretion have been proposed by Begelman 2001). Nevertheless, this does not alter our conclusion that 
stellar capture provides sufficient matter for maintaining an accretion rate at least at the Eddington level while the black hole mass is below the relation (24). For example, if we assume that a thin disk with fixed $\dot{m}$ is maintained while the black hole mass is small (with the "excess" matter delivered by stars being expelled in an outflow), then we have $\Sigma\left(r_{1 c}\right) \propto M_{B H}^{4 / 5} / r_{1 c}^{3 / 5} \propto M_{B H}^{-2 / 5} t^{3 / 5}$, which combined with $\Sigma\left(r_{1 c}\right) \propto M_{B H} / t$ yields $t \propto M_{B H}^{7 / 8}$, so the timescale for delivery of a mass $M_{B H}$ to the disk is still an increasing function of $M_{B H}$.

Hence, capture of stars will replenish the gas disk at a rate fast enough to maintain an Eddington accretion rate (with $L \simeq L_{E d d}$ and $\epsilon \simeq 0.1$ ) while the black hole mass is less than in equation $(24)$ for $\dot{m} \simeq 10$. When this value of the black hole mass is reached, the disk may still continue to be fed at a decreasing value of $\dot{m}$, and the black hole mass can keep growing up to a value $M_{B H} \propto(\dot{m})^{-5 / 7}$, according to equation (24). This continued growth requires that star captures continue over the increasingly long timescale $t_{S} \propto \dot{m}^{-1}$.

The growth of the black hole may eventually stop due to three possible mechanisms. The first is the aging of the stellar population, which reduces the number of available stars for capture and the mean value of $M_{*}^{5 / 7} / v_{e *}^{20 / 7}$ in equation (24). The second is the depletion of the loss-cylinder as the post-starburst stellar system reaches equilibrium and the rate of orbital relaxation declines. The third is a transition of the gas accretion into the black hole from a thin, radiatively efficient disk into a hot, advection dominated accretion flow or inflow/outflow solution (e.g., Blandford \& Begelman 1999; Quataert 2003). This transition to a hot, thick flow may occur at a critical value of $\dot{m}$ as it decreases (Begelman \& Celotti 2004), and it implies a dramatic decline of the surface density which would immediately terminate the capture of stars and cause a sudden decrease in $\dot{m}$. Therefore, our model also suggests a mechanism by which quasars turn off, leaving behind a remnant black hole that will stay at a constant mass thereafter if there are no additional nuclear starbursts. This final black hole mass should therefore depend on the value of $\dot{m}$ at which the accretion ceases. If this value of $\dot{m}$ depended on $M_{B H}$, the slope of the $M_{B H}-\sigma_{c}$ relation would be altered.

There are a number of possible problems with the simple picture presented here that will need to be addressed in future work. The initial process by which the gas disk grows around a seed black hole, reaching the Eddington accretion rate out to a certain radius, needs to be examined more carefully. The structure of the accretion disk should also be thoroughly modified from that of the simple steady-state model in $\S 2.1$, due to the effects of the capture of stars in adding mass, energy and angular momentum to the disk. Finally, the process by which a quasar would turn off as the stellar capture rate decreases and the stellar population ages also needs to be studied. 


\section{Self-Gravity of the Accretion Disk}

One of the classic problems encountered by standard quasar accretion disk models is that if matter is transported from large radius, from any residual gas in the galaxy that is left over from star formation, the gas should form a thin disk and become gravitationally unstable to form stars while it is still very far from the black hole (Shlosman \& Begelman 1987). In this case, the result might not be a black hole fed by an accretion disk, but simply a dense inner disk of stars. The criterion for gravitational instability is obtained from Toomre's parameter, $Q=\left(c_{s} \Omega\right) /(\pi G \Sigma)$, which using equations (8) and (9) can be expressed as (see also the Appendix in Goodman 2003)

$$
Q=\frac{45^{\frac{3}{10}}}{\pi^{\frac{9}{10}} 2^{\frac{27}{20}}} \dot{m}^{-\frac{2}{5}} \hat{\kappa}^{\frac{3}{10}} \beta^{\frac{7 b-12}{10}}\left(\frac{m_{P l}}{\mu m_{p}}\right)^{\frac{6}{5}}\left(\frac{m_{P l}}{M_{B H}}\right)^{\frac{13}{10}}\left[\frac{\alpha_{e}^{2} \alpha m_{P l}^{3}(1+X)}{m_{p} m_{e}^{2}}\right]^{\frac{7}{10}}\left(\frac{R_{S}}{r}\right)^{\frac{27}{20}}
$$

or in terms of fiducial values,

$$
Q=0.33 \dot{m}^{-\frac{2}{5}} \hat{\kappa}^{\frac{3}{10}} \beta^{\frac{7 b-12}{10}} M_{8}^{-\frac{13}{10}} \alpha_{-2}^{\frac{7}{10}}\left(\frac{10^{3} R_{S}}{r}\right)^{\frac{27}{20}}
$$

Contours of $Q=1$ are shown in Figure 1 (for $\dot{m}=10, \alpha_{-2}=1$ ). To the left of the contour, $Q>1$ and the disk is stable, while to the right the disk is gravitationally unstable. Figure 1 shows that the region of the disk within $r_{1 c}$ is mostly stable, except for large black hole masses for which the disk may be marginally unstable close to $r_{1 c}$. The effect of the stars crossing the disk would probably be important here in increasing the disk temperature and stabilizing it. Hence, the model proposed here may solve the problem of instability in quasar accretion disks at large radius, simply because most of the matter is delivered to the disk at small radius. Future work to calculate the disk structure including the effects of the plunging stars and possible extensions of the accretion disk to large radius with matter from stellar winds will be needed to examine this question in more detail.

\section{The Fate of the Captured Stars}

The orbits of the stars plunging through the disk are gradually circularized and brought into the disk plane in successive crossings. At every crossing, a fraction of the star might be stripped from its outer layers. If this mass loss were large enough stars could be destroyed before they merge into the disk.

Considering a typical case, for $M_{B H}=10^{8} M_{\odot}, \dot{m}=10, \alpha=10^{-2}$, and solar-type stars, a star crossing the disk at $r_{1 c} \simeq 500 R_{S}$ [eq. (26)] encounters a surface density $\Sigma \sim$ 
$5 \times 10^{6} \mathrm{~g} \mathrm{~cm}^{-2}$ with midplane temperature $T \sim 2 \times 10^{5} \mathrm{~K}, \beta \sim 0.4$, and $c_{s} \sim 70 \mathrm{~km} \mathrm{~s}^{-1}$ (as obtained from $\S 2.1$ ). The disk scaleheight is $H=c_{s} \Omega \sim 6 \mathrm{AU}$, and at a velocity $v \sim 10^{4} \mathrm{~km} \mathrm{~s}^{-1}$ the star takes a few days to cross the disk. The midplane disk density at $r_{1 c}$ is $\rho \sim \Sigma /(2 H) \sim 10^{-7.5} \mathrm{~g} \mathrm{~cm}^{-3}$. The ram pressure exerted by the disk gas on the star is $\rho v^{2} \sim 10^{10.5} \mathrm{erg} \mathrm{cm}^{-3}$. In a star like the Sun, this same pressure occurs at a radius $0.97 R_{*}$, which corresponds to a mass $M_{\text {out }} \sim 10^{-4.5} M_{*}$ outside this radius. However, most of this mass should simply be pushed against the star surface to higher pressure by the shock generated by the disk wind, and will not be lost. The disk material will be heated to $\sim 10^{9}$ $\mathrm{K}$ when it encounters the shock, and will then flow around the star. Any stellar mass loss during the disk passage depends on the heat conduction and irradiation from the post-shock disk material into the stellar surface, and any turbulent instabilities that may mix stellar matter into the disk wind. After the disk passage, the heated material in the stellar surface would cool back to equilibrium on a short timescale compared to the orbital period. We therefore expect that the mass loss of the plunging stars will be negligibly small, modulo the uncertain mixing processes of matter in the stellar surface and the post-shock disk wind that might act to destroy stars before they merge into the disk (see also Goodman \& Tan 2003).

Assuming that the stars survive, once embedded inside the disk they may create gaps around them if they are sufficiently massive, or they may accrete gas from the disk (Syer et al. 1991, Artymowicz et al. 1993). Stars might also evaporate into the disk instead of accreting if the midplane temperature is high enough. In any case, eventually the stars will be mostly destroyed and dissolved into the gaseous disk. That this must be the case can easily be seen by considering what would happen if a large fraction of the disk mass were in the form of stars of $M_{*} \simeq 1 M_{\odot}$. For typical parameters $M_{8}=1, r=500 R_{S}=1000 \mathrm{AU}$, and $\Sigma=10^{6.5} \mathrm{~g} \mathrm{~cm}^{-2}$, stars would have to occupy a fraction of the disk area of at least $10^{-5}$ (given their typical surface density $\Sigma_{*} \sim 10^{11} \mathrm{~g} \mathrm{~cm}^{-2}$ ), so in a ring of width equal to the solar diameter there would be about 10 stars. These numbers become even greater for more massive stars because their surface density is lower. This makes it clear that collisions and scatterings among stars would be frequent, and they would soon lead to coalescence and destruction of the stars. If a star is on a slightly eccentric orbit (either because the orbit has not yet fully circularized after capture or because it has been scattered by other stars), a collision can take place at a sufficiently high relative velocity to cause the dissolution of the stars rather than coalescence. If stars can coalesce repeatedly, they will become very massive and lose a lot of their mass in winds (see Goodman \& Tan 2003 for a discussion of the possible presence of supermassive stars in accretion disks). Very massive stars are also highly unstable because their internal energy is almost balanced by their negative gravitational energy (since they are polytropes with adiabatic index very close to $4 / 3$ ), so collisions with other stars would not have to 
occur at very high velocities to produce large amounts of mass loss. It therefore appears inevitable that a large fraction of the mass in the stars will be dissolved into the disk, and can eventually be accreted onto the black hole with high radiative efficiency.

\section{The Angular Momentum Problem}

The idea presented in this paper is that most of the matter in the black hole accretion disk comes from stars that were originally in orbits with radius much larger than the size of the disk. These stars were perturbed into highly eccentric orbits and captured by the disk, and were embedded into the disk after repeated disk crossings in which their orbital energy was dissipated.

If we imagine that the accretion disk has to be initially formed from the matter of these plunging stars (starting from an initial small disk which can grow in mass from captured stars until it reaches a steady-state structure), we are faced with the problem of how the disk has acquired its angular momentum. The average angular momentum of the captured stars is far too small to make a disk. Although the specific angular momentum of an individual plunging star is of the same order as the specific angular momentum of the disk gas at the radius $r_{1 c}$ where the star is captured, the direction of the angular momentum of each star is basically random. Even if the stellar system around the black hole is rotating (implying a phase space density of stars that depends on the direction of the orbital angular momentum), the stars captured by the disk are coming from a very narrow loss-cylinder, and the variation of the phase space density over this narrow region is negligible. Thus, if the disk is made solely by these stars, the final specific angular momentum of the captured matter is reduced by the square root of the number of stars that have contributed to the disk mass. In other words, the radius of the disk that could be made by the matter from these stars is reduced relative to the capture radius $r_{1 c}$ by a factor equal to the number of captured stars, which would make the disk smaller than the Schwarzschild radius. Clearly, a disk around the black hole cannot be made by stars captured from orbits with random orientations.

This problem is, however, much less severe if the disk is considered to be in place initially, and the plunging stars only have to maintain the disk in a steady state. If we imagine that the disk is truncated at a radius $\sim r_{1 c}$, where most of the plunging stars are incorporated to the disk, then the angular momentum that flows out of the disk and into the black hole is very small (because the radius of the innermost stable circular orbit from which matter is accreted to the black hole is much smaller than $r_{1 c}$ ), and the angular momentum that flows into the disk from the plunging stars is also very small. Hence, the disk angular momentum can basically be preserved: as matter flows inwards in the disk, 
it releases its angular momentum by viscosity processes towards the outer disk, and this angular momentum is constantly being absorbed by the matter added from plunging stars. With this simplified description, it can be argued that the scaling of the disk surface density profile with black hole mass and radius in equation (9) would essentially be preserved when the energy and angular momentum added to the disk by the plunging stars are included selfconsistently, except that the disk is truncated around radius $r_{1 c}$ where most of the matter from stars is added.

This greatly alleviates, but does not completely solve, the angular momentum problem, because there is still a small amount of angular momentum that is transferred to the black hole as the accretion proceeds. Moreover, the problem may be made worse because the stars that have angular momentum opposite to that of the disk should have a higher probability of capture owing to their larger relative velocity with respect to the disk material. This implies that the captured stars would actually carry a net average angular momentum to the disk but with opposite direction. A full solution of the problem may require the addition of some matter to the disk with high specific angular momentum from large radius. The disk would not need to be completely cut off at radius larger than $r_{1 c}$, but it could continue at a lower surface density. The outer-disk matter with high angular momentum can originate from gas that is left over from star formation or has been expelled in winds from evolved stars and supernova explosions, and its angular momentum can come from a small rotation rate of the stellar system around the black hole. Note that the matter accreting from a disk at radius larger than $r_{1 c}$ needs to be only a small fraction of the total, with most of the mass coming from plunging stars (because only a small fraction of the disk angular momentum needs to be replenished every time its mass is replaced within $r_{1 c}$ ), thereby preserving the explanation for the $M-\sigma$ relation we have proposed in this paper. As for the problem that stars moving on orbits with angular momentum opposite to that of the disk are more likely to be captured than stars moving in the same sense of rotation as the disk, we note that while stars lose most of their orbital energy during disk crossings at the smallest pericenter, the exchange of angular momentum with the disk is dominated by crossings at large radius for a disk surface density profile $\Sigma(r) \propto r^{-3 / 5}$. This may help solve the problem in a disk extending to $r \gg r_{1 c}$ with a small fraction of the accretion rate contributed by stellar captures.

The total angular momentum of the accretion disk within $r_{1 c}$ might, in fact, be selfregulated by the presence of an $\mathrm{AGN}$ wind. If random fluctuations in the number of plunging stars coming in with different angular momenta cause the disk to lose a lot of angular momentum at some point, the natural response is that as the disk shrinks the accretion rate is increased and a strong AGN wind results when the luminosity becomes too close to the Eddington value. Some of the ejected material might then fall back into the disk at larger radius, mixing with gas of high specific angular momentum and dragging some of that gas 
to smaller radius.

Another mechanism that may cause a mixing of material of different specific angular momentum is the lifting of gas from the disk when stars go through it. This was described by Zurek et al. (1994, 1996), who suggested that the resulting star tails and debris might be the origin of the broad emission lines in AGN.

\section{Discussion}

Several models have been suggested that can explain the $M_{B H}-\sigma_{*}$ relation. These fall broadly into two distinct classes: models in which the growth of the black hole is limited by either radiative or mechanical feedback from the active nucleus (e.g., Silk \& Rees 1998; Haehnelt, Natarajan, \& Rees 1998; Blandford \& Begelman 1999, 2004; King 2003; Wyithe \& Loeb 2003; Norman et al. 2004) and models in which the matter available in the bulge in stars or gas determine the feeding of the central black hole (e.g., Zhao, Haehnelt, \& Rees 2002; Merritt \& Poon 2004). The first class of models face the difficulty of explaining how enough material accretes from the large distances required down to the central engine via an accretion disk, as we describe in more detail below. Moreover, it is not clear how an outflow can be sustained over the time required to entirely expel all the matter that could potentially be fed to the black hole at a future time, out to a large distance from the host galaxy, without at the same affecting the accretion disk much closer to the black hole, which is energizing the outflow. The second class of models (to which our model belongs), when using stars to grow the black holes, have been faced up to now by the key problem that they do not account for the high-redshift quasars and that the black holes grow only on very long timescales.

The model introduced in this paper proposes that the principal mechanism by which accretion disks around quasars acquire their mass is from stars that plunge through the disk. The increased cross section for capturing a star by the disk, compared to a direct capture by the black hole, allows black holes to be fed at a rapid rate, thereby avoiding the timescale

problems encountered by previous models in this class. Furthermore, because the stellar matter is dissolved into a thin disk before falling into the black hole, and then it accretes at high radiative efficiency, quasars are naturally accounted for as the main mechanism by which black holes grew. Accretion disks around quasars are predicted to be much smaller than previously believed (although a small fraction of the accreted matter may be carried in as gas from large radius), thereby avoiding the disk gravitational instability problem. The predicted $M_{B H}-\sigma_{*}$ relation is consistent with observations, and is related by our model to fundamental parameters of the accretion disk and properties of the stellar population. 
As pointed out previously by Ostriker (1983), Syer et al. (1991), and others, it is inevitable that some stars will be captured through this process because the dense nuclear regions of galaxies must always contain stars. The question is whether or not star captures will occur at the high rate necessary to feed the disk and account for the final black hole mass. We have shown that the answer to this question is affirmative provided that two crucial assumptions are satisfied: first, that nuclear starbursts produce a roughly isothermal initial density profile of stars with a velocity dispersion $\sigma_{c}$ similar to that of the whole spheroidal component (with any core radius in the stellar distribution being smaller than the zone of influence of the final black hole, $r_{c}$ ); and second, that the rate at which stars are brought into a nearly radial orbit due to dynamical relaxation and the triaxiality of the potential (which can result in a substantial fraction of stochastic orbits) is sufficient to keep the loss-cylinder full. These assumptions may turn out to be wrong, and if this is the case other explanations will need to be found for how enough gas is transported to the inner accretion disk in quasars without turning to stars along the way and for the $M_{B H}-\sigma_{*}$ relation. But if the assumptions are correct, our model provides a unified solution of these apparently unrelated problems.

The detailed predicted form of the $M_{B H}-\sigma_{*}$ correlation is still subject to theoretical uncertainties that will need to be carefully analyzed as the model we have introduced here is developed further. The surface density profile of the disk, which is the main property that determines the form of the $M_{B H}-\sigma_{c}$ correlation, is affected by the viscosity mechanism that causes the disk to accrete to the black hole, by the accretion rate of the quasar disk, and by the heating and addition of angular momentum to the disk associated with the plunging stars and their transformation inside the disk. Nevertheless, the simple analysis presented in this paper based on a disk with no heat source except viscous dissipation and no redistribution of angular momentum (plus the argument we give in $\S 5$ that the effects of the plunging stars are likely to be a truncation of the disk around radius $r_{1 c}$, maintaining the basic scalings of the disk surface density profile with mass, radius and accretion rate) suggests that it is plausible that this model can account for the observed correlation.

This plunging star model has the added benefit of providing a simple way to deliver the large mass of the black hole to a very small disk around it. The difficulty of explaining how nuclear black holes have grown so big in the standard (but hypothetical) scenario whereby gas reaches the center by accretion through a disk extending to large radius should not be overlooked (e.g., Begelman 2003). In fact, taking as a typical example a black hole with $M_{B H}=10^{8} M_{\odot}$ in a galaxy with $\sigma_{*}=200 \mathrm{~km} \mathrm{~s}^{-1}$ and stellar mass $M_{b} \sim 10^{11} M_{\odot}$ within $5 \mathrm{kpc}$ of the center, the radius initially containing a mass of $10^{8} M_{\odot}$ is $5 \mathrm{pc}$ for a singular isothermal profile (and even larger for shallower profiles). It is very difficult to see how all this mass could form a gaseous disk and then accrete inwards by three orders of magnitude in radius over the lifetime of the quasar, without turning into stars. Observationally, we see 
that star formation is a universal phenomenon taking place in every galaxy that contains cold gas above some critical surface density threshold (e.g., Kennicutt 1989; Martin \& Kennicutt 2001), and that cold gas does not migrate very much in radius before it turns to stars. And yet, in the standard quasar model one is forced to assume that the same thing does not happen in the innermost parts of galaxies, and that cold gas is efficiently transported to the nucleus. Our model solves this problem by proposing that all this gas does indeed turn into stars, and then the stars are captured by the disk at small radius. In this way, the disk gravitational instability problem is likely also solved. The disk can still extend to radius much larger than $r_{1 c}$ from additional gaseous material left over from star formation and expelled by evolved stars (which could be a source of angular momentum for the disk), but with a much lower accretion rate than in the inner disk supplied by plunging stars. The low surface density of this outer disk, plus the additional heating source provided by plunging stars, can help prevent star formation at large radius (see Sirko \& Goodman 2003 for models of marginally self-gravitating disks).

Norman \& Scoville (1988) also suggested a model in which quasars are fueled by stars after a nuclear starburst, but in their case all the matter is expelled by evolved stars. Their model still does not account for why the gas present in this nuclear region stops forming stars at some point and starts accreting into the black hole instead. Our model brings the stellar matter in the central cluster close to the black hole directly, and provides a natural black hole mass at which this process should stop.

What other observational predictions can our model make? Clearly the structure of the accretion disk should be modified by the capture of stars, probably showing some characteristic feature in the surface density and temperature profiles at the capture radius. A clear test of this scenario will require much more advanced theoretical modeling than we have done here to obtain predictions for the disk profiles, and observations that can resolve the continuum emission of the disk. The addition of angular momentum to the central parts of the disk by plunging stars coming in along random orbits may generate warped inner disks with a characteristic variability timescale that could have observable consequences. The star tails generated by the plunging stars may give rise to the broad emission line region, as discussed by Zurek et al. (1994, 1996). Hydrodynamical simulations may be necessary here to make any predictions that could be confronted with observational tests, which could perhaps suggest some diagnostic for the rate at which stellar collisions with the disk occur, testing if the rate is as high as required by our model.

The clearest prediction of our model is that the quasar phenomenon must take place in the context of nuclear starbursts. Only a very compact starburst can provide the high density of stars in the nucleus that is necessary to feed the accretion disk. Quasars may always follow 
an initial stage of growth within a highly obscured compact starburst region, which becomes highly ionized and transparent only after the quasar has reached a high luminosity and cleared the surrounding dust. The light contribution from the starburst around the quasar will be difficult to discern, because the total mass of the starburst that forms the plunging stars within radius $r_{c}$ is comparable to the total mass that will be accreted by the black hole, and the radiative efficiency of gas accretion to the black hole is much higher than the efficiency of nuclear burning in stars. The most straightforward observational test of our model would be to image the central few parsecs around a luminous quasar and see if the population of plunging stars is there, but the high resolution required and the small amount of light coming from the stars compared to the quasar may make this a difficult challenge. In our model, the size of the nuclear starburst from which stars are captured is $r_{c} \sim 5 \mathrm{pc}\left(M_{B H} / 10^{8} M_{\odot}\right)^{8 / 15}$, much smaller than the highest resolution images available from nearby Seyfert galaxies (Pogge \& Martini 2002).

The high stellar density of the required nuclear starbursts does not need to be preserved at the present time, long after the quasar is dead. Several dynamical phenomena can reduce the central stellar density: if massive stars are dominant in nuclear starbursts, only the light from the small fraction of mass that formed low-mass stars would be visible at present. Moreover, a large fraction of the initial stellar mass would have been lost in stellar winds and supernovae, resulting in adiabatic expansion of the remaining stellar population. Mergers of galaxies harboring nuclear black holes from old quasars will lead to the merger of black holes, which eject the stars near the center and create a wide core in the stellar profile (Milosavljević \& Merritt 2001). Diverse merger histories in different galaxies may introduce the large variability observed in the slope of the innermost stellar profiles in elliptical galaxies (Faber et al. 1997; Balcells, Graham, \& Peletier 2004). Even though our model predicts that $M_{B H}$ is correlated with the velocity dispersion of the nuclear starburst at the small radius, $r_{c}$, from which most of the captured stars come, the correlation with the velocity dispersion can remain a tight one out to much larger radius if nuclear starbursts tend to form with a universal density profile close to isothermal. Then, after the inner part of the stellar distribution is altered by repeated galaxy and black hole mergers, the remaining present correlation would be tightest when expressed in terms of the velocity dispersion at large radius.

The model also predicts that the $M_{B H}-\sigma_{*}$ relation should be independent of redshift, since it is imprinted at the time the quasars formed, except for the effects of galaxy mergers and the passive evolution of the stellar population in changing the velocity dispersion. The small scatter of the relation is easier to understand if galaxy mergers shift the black hole mass and velocity dispersion of galaxies in a direction approximately parallel to the $M_{B H}-\sigma_{*}$ relation (as seems to be implied by the Faber-Jackson relation). This suggests that the 
$M_{B H}-\sigma_{*}$ relation may remain unmodified by mergers and should then be close to constant with redshift.

Another prediction of our model is that not all active galactic nuclei should lie on the same $M_{B H}-\sigma_{*}$ relation as the inactive black holes, but they should deviate from it in a way that depends on the $L / L_{E d d}$ ratio. When a black hole becomes active after a nuclear starburst has occurred, its mass may initially be small and the supply of fuel from plunging stars will be more than sufficient to maintain an Eddington luminosity, but as the black hole grows in mass and approaches the final $M_{B H}-\sigma_{*}$ relation, the ratio $L / L_{E d d}$ has to decrease if the quasar is to be sustained (as discussed in §2.5). Hence, active nuclei with $L \simeq L_{E d d}$ should lie below the relation, and they should gradually come closer to the relation as $L / L_{E d d}$ decreases towards a minimum value at which the nuclear activity typically ceases, with $M_{B H} \propto\left(L / L_{E d d}\right)^{-5 / 7} \sigma_{*}^{30 / 7}$ if the radiative efficiency $\epsilon$ is constant (see eq. [24]). Recent data on Narrow line Seyfert I galaxies suggest a correlation that is roughly along these lines (Mathur et al. 2001; Grupe \& Mathur 2004; Mathur \& Grupe 2004).

Finally, the $M_{B H}-\sigma_{*}$ relation should break down at a black hole mass where the mass of the accretion disk becomes comparable to that of a star. When that happens, the timescale for the disk to accrete onto the black hole should be comparable to the mean time to capture one star, so the disk can disappear when, by chance, no star is captured over a long enough period of time. Approximating the mass of the disk as $\pi r_{1 c}^{2} \Sigma\left(r_{1 c}\right)$, and from equations (12) and (26), we find that the mass of the disk is $1 M_{\odot}$ at $M_{B H} \sim 10^{4.5} M_{\odot}$. This shows that the mechanism we have presented here cannot continue to operate below this mass.

In summary, we have presented a novel mechanism for the formation of nuclear black holes in the centers of galaxies through the capture of stars by a gaseous accretion disk. Our model provides a physical connection between the stellar populations of bulges and black hole growth and reproduces the observed $M_{B H}-\sigma_{*}$ relation for reasonable input assumptions as a natural consequence.

We are grateful to Martin Haehnelt, Jordi Isern, David Merritt, Martin Rees, Scott Tremaine, and David Weinberg for many insightful discussions. JAK acknowledges the support of a university fellowship at Ohio State. This work was supported in part by grant NSF-0098515. 


\section{REFERENCES}

Aller, M. C., \& Richstone, D. 2002, AJ, 124, 3035

Artymowicz, P., Lin, D. N. C., \& Wampler, E. J. 1993, ApJ, 409, 592

Balcells, M., Graham, A. W., \& Peletier, R. C. 2004, submitted to ApJ (astro-ph/0404379)

Barger, A. J., Cowie, L. L., Bautz, M. W., Brandt, W. N., Garmire, G. P., Hornschemeier, A. E., Ivison, R. J., \& Owen, F. N. 2001, AJ, 122, 2177

Becker, R. H., et al. 2001, AJ, 122, 2850

Begelman, M. C. 2001, ApJ, 551, 897

Begelman, M. C. 2003, in Active Galactic Nuclei: from Central Engine to Host Galaxy, eds. S. Collin, F. Combs, I. Shlosman (ASP Conference Series 290), p. 23

Begelman, M. C., \& Celotti, A. 2004, MNRAS, in press (astro-ph/0406607)

Blandford, R. D., \& Begelman, M. C. 1999, MNRAS, 303, L1

Boyle, B. J., Shanks, T., Croom, S. M., Smith, R. J., Miller, L., Loaring, N., \& Heymans, C. 2000, MNRAS, 317, 1014

Boyle, B. J., Shanks, T., \& Peterson, B. A. 1988, MNRAS, 235, 935

Colgate, S. A. 1970, Nat, 225, 247

Faber, S. M., et al. 1997, AJ, 114, 1771

Gebhardt, K., et al. 2000, ApJ, 539, L13

Goodman, J. 2003, MNRAS, 339, 937

Grupe, D., \& Mathur, S. 2004, ApJL, 606, 41

Haehnelt, M. G. 2003, Carnegie Observatories Astrophysics Series, Vol. 1: Coevolution of Black Holes and Galaxies, ed. L. Ho (Cambridge: Cambridge Univ. Press), in press (astro-ph/0307378)

Haehnelt, M, \& Kauffmann, G. 2000, MNRAS, 318, L35

Heckman, T., Kauffmann, G., Brinchmann, J., Charlot, S., Tremonti, C., \& White, S. D. M. 2004, ApJ, in press (astro-ph/0406218) 
Kauffmann, G., \& Haehnelt, M. 2000, MNRAS, 311, 576

Kennicutt, R. C. 1989, ApJ, 344, 685

King, A. 2003, ApJL, 596, L27

Kormendy, J., \& Richstone, D. 1995, ARA\&A, 33, 581

Lynden-Bell, D. 1969, Nat, 223, 690

Magorrian, J., et al. 1998, AJ, 115, 2285

Martin, C. L., \& Kennicutt, R. C. 2001, ApJ, 555, 301

Mathur, S., \& Grupe, D. 2004, submitted to A\&A (astro-ph/0407512)

Mathur, S., Kuraszkiewicz, J., \& Czerny, B. 2001, NewA, 6, 321

Merritt, D., \& Ferrarese, L. 2000, ApJ, 539, L9

Merritt, D., \& Ferrarese, L. 2001, ApJ, 547, 140

Merritt, D., \& Poon, M. Y. 2003, submitted (astro-ph/0302296)

Milosavljević, M., \& Merritt, D. 2001, ApJ, 563, 34

Norman, C., \& Scoville, N. 1988, ApJ, 332, 124

Ohsuga, K., Mineshige, S., Mori, M., \& Umemura, M. 2002, ApJ, 574, 315

Ostriker, J. P. 1983, ApJ, 273, 99

Quataert, E. 2003, Astron. Nachr., 324, 3 (astro-ph/0304099)

Quinlan, G. D. 1996, NewA, 1, 35

Pogge, R. W., \& Martini, P. 2002, ApJ, 569, 624

Pringle, J. E. 1981, ARA\& A, 19, 137

Richstone, D., et al. 1998, Nat, 395, 14

Salpeter, E. E. 1964, ApJ, 140, 796

Shakura, N. I., \& Sunyaev, R. A. 1973, A\& A, 24, 337

Shlosman, I., \& Begelman, M. C. 1987, Nat, 329, 810 
Sirko, E., \& Goodman, J. 2003, MNRAS, 341, 501

Soltan, A. 1982, MNRAS, 200, 115

Syer, D., Clarke, C. J., \& Rees, M. J. 1991, MNRAS, 250, 505

Tremaine, S., et al. 2002, ApJ, 574, 740

Wang, J.-M., Szuszkiewicz, E., Lu, F. J., \& Zhou, Y. Y. 1999, ApJ, 522, 839

Wheeler, J. C., Lecar, M., \& McKee, C. F. 1975, ApJ, 200, 145

Wyithe, J. S. B., \& Loeb, A. 2003, ApJ, 595, 614

Yu, Q., \& Tremaine, S. 2002, MNRAS, 335, 965

Zhao, H., Haehnelt, M. G., \& Rees, M. J. 2002, NewA, 7, 385

Zurek, W. H., Siemiginowska, A., \& Colgate, S. A. 1994, ApJ, 434, 46

Zurek, W. H., Siemiginowska, A., \& Colgate, S. A. 1996, ApJ, 470, 652 

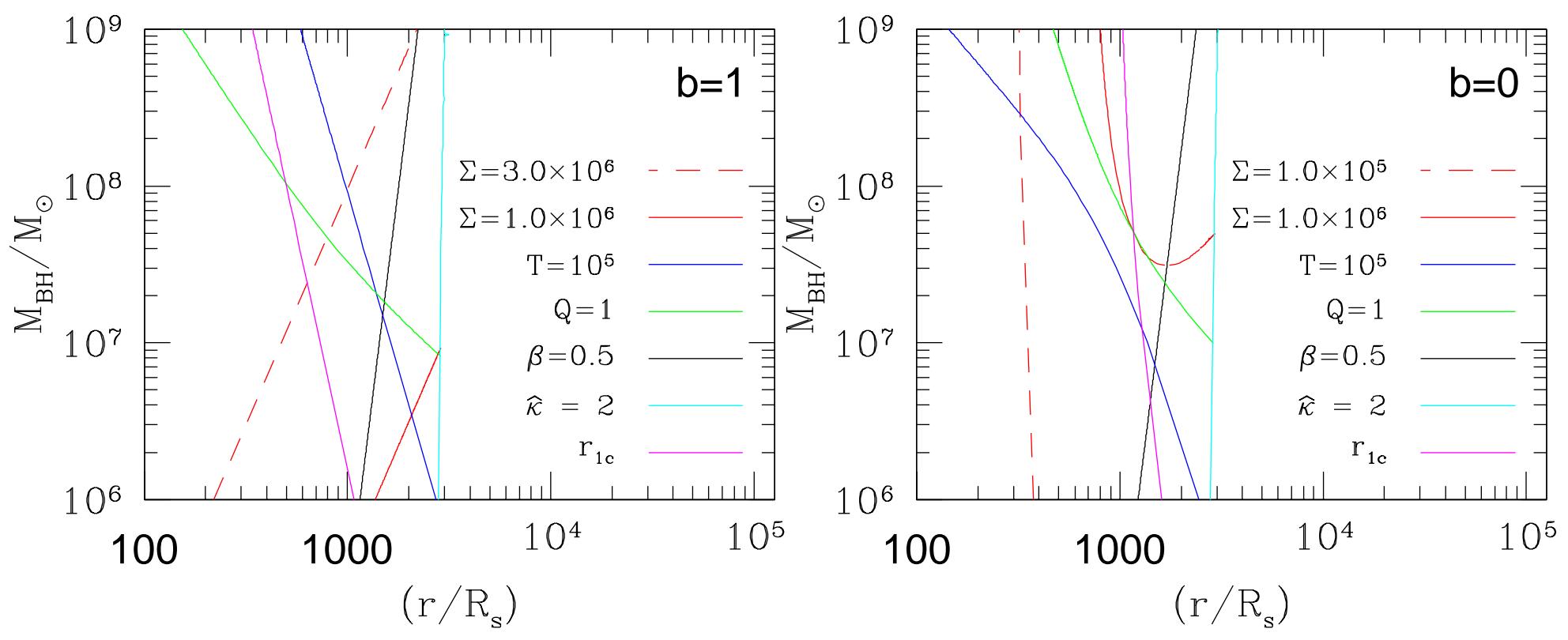

Fig. 1.- Contours of accretion disk parameters $\Sigma\left(\mathrm{g} \mathrm{cm}^{-2}\right), \mathrm{T}(\mathrm{K}), \mathrm{Q}, \beta, \hat{\kappa}$, and $\mathrm{r}_{1 c} / \mathrm{R}_{\mathrm{s}}$ as indicated in each panel. Left: Contours for the case of $b=1$, that is, the disk viscosity is proportional to the gas pressure. Right: Contours for the case of $b=0$, that is, the disk viscosity is proportional to the total pressure. All contours are computed for $M_{*} / M_{\odot}=1$, $\alpha_{-2}=1, \dot{m}=10$, and $\hat{\kappa}=1$. We truncate curves to the right of the $\hat{\kappa}=2$ contour, as they are no longer valid in this region. 